\section{Prix Nobel de Médecine 2016}

\section{Autophagie}

Yoshinori Ohsumi

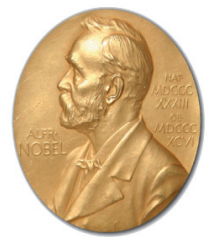

(C) https://www.nobelprize.org

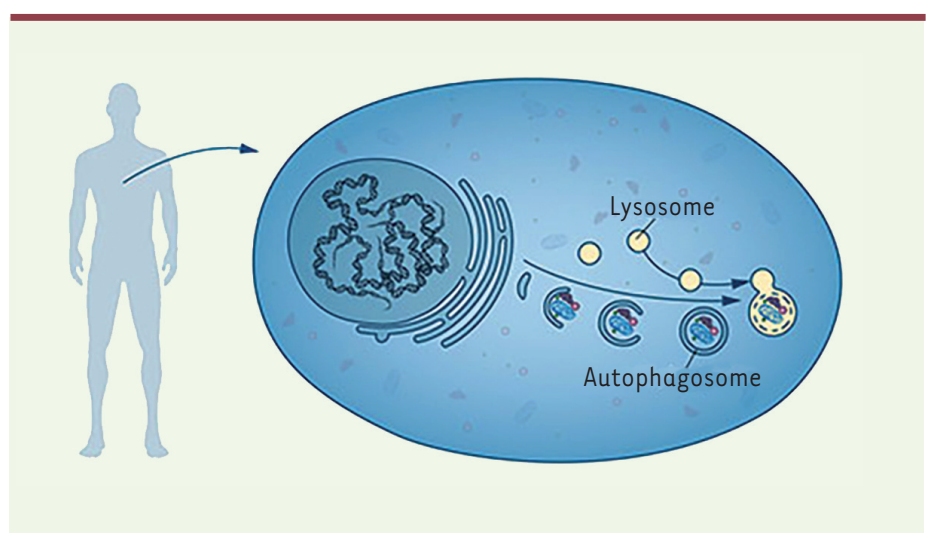

Autophagie : dégradation et recyclage (๔ https://www.nobelprize.org)

Renaud Legouis (Autophagie et développement)

Lorenzo Galluzzi (Autophagie et vieillissement)

Sophie Lotersztajn et Philippe Gual (Autophagie et foie)

Maryam Mehrpour (Autophagie et homéostasie du fer)

Valérie Pierrefite-Carle (Autophagie et os)

Benjamin Dehay (Autophagie et cerveau)

Nicolas Pallet (Autophagie et rein)

Chantal Boulanger et Pierre-Emmanuel Rautou (Autophagie, cœur et vaisseaux)

Béatrice Romagnolo (Autophagie et intestin)

Juan lovanna (Autophagie et pancréas)

Sophie Lanone (Autophagie et poumon - Nanoparticules)

Patricia Boya (Autophagie et vision)

Mathias Faure (Autophagie et inflammation)

Isabelle Vergne, Frank Lafont, Audrey Esclatine, Martine Biard-

Piechaczyk et Lucile Espert (Autophagie et maladies infectieuses)

Sylviane Muller (Autophagie et maladies auto-immunes)

Sophie Pattingre, Mojgan Mergny, Sylvie Giuriato et Carine Joffre

(Autophagie et cancer)

Patrick Auberger (Autophagie et enjeux thérapeutiques) 